\title{
A Review: Biologics in Management of Psoriasis
}

\author{
Anupriya Kapoor ${ }^{1}$, Ajay Kumar ${ }^{1}$, Arpit Katiyar ${ }^{2}$ and Shashi Kiran Misra ${ }^{1 *}$ \\ ${ }^{1}$ School of Pharmaceutical Sciences, CSJM University, Kanpur, India \\ ${ }^{2}$ Sai Meer College of Pharmacy, Kannauj, India \\ *Corresponding Author: Shashi Kiran Misra, School of pharmaceutical sciences, \\ CSJM University, Kanpur, India.
}

Received: August 08, 2021

Published: September 23, 2021

(C) All rights are reserved by Shashi Kiran

Misra., et al.

\begin{abstract}
Biologic drugs also referred to as biologic response modifiers are powerful medications that comprise small fractions of components like sugars, proteins, or DNA. These drugs include a wide variety of products that are derived from all sorts of living sources including mammals, birds, insects, plants, and even micro-organisms. They offer a new era in the treatment of diseases as they possess the capability to change the manner of operation of biological intracellular and cellular actions. The developments in the field of biologics have added new dimensions in the treatment of dermatological issues. They have emerged as appropriate alternatives for conventional therapies especially in instances where they fail to respond to patient's needs. The therapy including the use of biologics based on a treatment approach that involves the interaction with specific underlying components of the immune system. The review article highlights the biologics used in the treatment of psoriasis since it is a worldwide dermatological problem causing significant pathological and psychological complications.
\end{abstract}

Keywords: Biologics; Psoriasis; Adalimumab; Etanercept; Secukinumab; Ixekizumab

\section{Introduction}

Over the past two decades, many treatment advancements have occurred, that have paved new paths for the treatment of Psoriasis. Biologics have proved to be a better alternative for the treatment of psoriasis especially in cases when the general medications fail to elicit desired responses. With the progress in the field of biologics, the treatment of psoriasis has undergone dramatic changes due to its high efficacy and safety. The advent in the development of biologics has also led to a better understanding of the pathogenesis of psoriasis.

Psoriasis is an autoimmune disorder that is characterized by the occurrence of scaly indurate erythema. The disease not only harms the health of the patient but also has an enormous effect on the quality of life of an individual. Although the disorder has a topical effect but is also found to be associated with systemic effects like psoriatic arthritis and mental disturbances [1]. A variety of biologics are available for the management of psoriasis however certain elements like rapidity in the onset of action, biological halflife, long-term efficacy, and safety profile are characteristics that are to be considered in designing the regimen to suit individual patient's needs [2].

\section{Classification of biologics}

The biologics used for the treatment of psoriasis are classified into various categories depending upon the target to show inhibition activity. Table 1 provides the classification of biologics with suitable examples.

How do biologics differ from synthetic drugs? [3,4]

Biologics are drugs that are derived from living cells and have structurally complex proteins that are not feasible to be chemically manufactured. On the other hand, synthetic drugs are made from the chemical process and have a simple chemical structure. 


\begin{tabular}{|c|c|}
\hline The target site of biologics & Drugs \\
\hline TNF- $\alpha$ inhibitors & $\begin{array}{r}\text { Adalimumab, Certolizumab-pegol, } \\
\text { Etanercept }\end{array}$ \\
\hline IL-12/23 inhibitor & Ustekinumab \\
\hline IL-23 inhibitors & Guselkumab, Risankizumab \\
\hline IL-17 inhibitors & Secukinumab, Ixekizumab \\
\hline CTLA4-Ig & Abatacept \\
\hline
\end{tabular}

Table 1: Classification of biologics with suitable examples.

Biologics offer vivid advantages over synthetic drugs. Biologics are target-specific and the side effects observed are related to exaggerated pharmacological effects, on contrary, the synthetic molecule expresses harmful non-target effects. Table 2 depicts the differences between biologics and synthetic drugs.

\begin{tabular}{|c|c|c|}
\hline Property & Synthetic drug & Biologic drug \\
\hline Size & Small molecule & Large molecule \\
\hline Molecular weight & $<500-900$ daltons & $>140000$ daltons \\
\hline $\begin{array}{l}\text { Manufacturing } \\
\text { process }\end{array}$ & $\begin{array}{c}\text { Through chemical } \\
\text { synthesis }\end{array}$ & $\begin{array}{c}\text { Through recombi- } \\
\text { nant DNA technol- } \\
\text { ogy }\end{array}$ \\
\hline Reproducing ability & $\begin{array}{l}\text { The predictable } \\
\text { chemical process } \\
\text { leads to the manu- } \\
\text { facturing of identi- } \\
\text { cal copies }\end{array}$ & $\begin{array}{l}\text { Specialized biologi- } \\
\text { cal process, made } \\
\text { in living cells }\end{array}$ \\
\hline Complexity & Simple molecule & Complex molecule \\
\hline $\begin{array}{c}\text { Route of adminis- } \\
\text { tration }\end{array}$ & $\begin{array}{c}\text { The preferred route } \\
\text { is oral and nasal }\end{array}$ & $\begin{array}{l}\text { The preferred } \\
\text { route is IV/ SC }\end{array}$ \\
\hline Permeability & High & Low \\
\hline Distribution & Easily distributed & $\begin{array}{c}\text { Limited distribu- } \\
\text { tion }\end{array}$ \\
\hline Immunogenicity & Absent & Present \\
\hline Stability & Mostly stable & $\begin{array}{l}\text { Sensitive to stor- } \\
\text { age and handling } \\
\text { conditions }\end{array}$ \\
\hline
\end{tabular}

Table 2: Differences between biologics and synthetic drugs.

The upsides of the usage of biologics

- $\quad$ Treatment regimens designed with the use of biologics will be cost-efficient in the future as compared to conventional drug therapy.
- The Same drug can be utilized for the treatment of multiple diseases.

- Biologics are associated with fewer side effects.

The downside of the usage of biologics [5]

Like synthetic drugs, biologics also possess certain side effects with the regimen. Some of the drawbacks associated with biologics are

- The biologics show allergic reactions, the most common of which include breathlessness, nausea, rash, itchiness, itchy eyes, etc.

- There are certain instances where there is the involvement of the central nervous system and cardiovascular system.

- $\quad$ Presence of lupus-like syndrome is also observed, which involves cell lysis and massive release of DNA.

- Suppression of the immune system is a common problem associated with the consumption of biologics.

- The usage of biologics also shows an increased risk of infections that includes respiratory tract infection, pneumonia, urinary tract infections, hepatitis $\mathrm{B}$, tuberculosis, histoplasmosis, etc.

- Opportunistic infections that are conferred with the usage of biologics include listeriosis, nocardiosis, etc.

- The most serious challenge of concern is the development of cancer due to the long-term utilization of biologics.

\section{Biologics in treatment of psoriasis}

Although the pharmaceutical market is flooded with multiple conventional options for the treatment of psoriasis that include a range of pills, creams, ointments, light therapy, etc. however in situations where conventional treatment does not work, biologics offer a refreshing path that offers an edge over conventional therapy. The drugs falling under the category of biologics offer their therapeutic benefit by blocking the cells and proteins that play a critical role in the pathogenesis of psoriasis. The general mechanism of action for different categories of biologics is compiled and depicted in figure 1. 


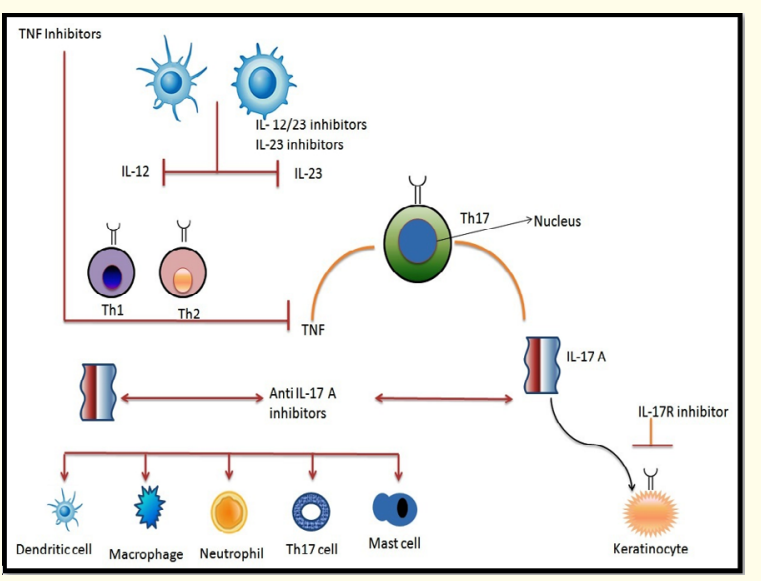

Figure 1: General mechanism of action for different categories of biologics.

\section{Adalimumab}

Sold under the brand name Humira is an anti-TNF monoclonal antibody that is used for the treatment of Crohn's disease, rheumatoid arthritis, ulcerative colitis, hidradenitis, psoriasis, psoriatic arthritis, etc. the drug got its approval by the FDA in the year 2002. The drug is given through subcutaneous injection. The mechanism of action of adalimumab involves the neutralization of TNF-alpha bioactivity by preventing the interaction of TNF-alpha with the cell surface TNF receptors. By this mechanism, adalimumab inhibits several TNF-alpha-induced events, ie, the release of serum cytokines (IL-6), acute phase reactants of inflammation, matrix metalloproteases, and other markers of cartilage and synovium turnover, and the expression of adhesion molecules responsible for leukocyte migration [6]. Due to the importance of TNF in host defense, one of the issues of prime adalimumab, is the increased risk of infections and malignancies $[7,8]$ and the most important infectious complication is the reactivation of tuberculosis [9].

\section{Abatacept}

Sold under the brand name Orencia was developed by Bristol-Myers Squibb and is licensed in the United States. The drug was approved by FDA in the year 2005 is given by route [10]. It is a modified antibody that is a fusion protein composed of the Fc region of the immunoglobulin IgG1 fused to the extracellular domain of CTLA-4. It is used to treat psoriatic arthritis and juvenile idiopathic arthritis $[11,12]$. The mechanism of action of Abatacept is based on the fact that it prevents antigen-presenting cells (APCs) from delivering the co-stimulatory signal. This prevents the T cells from being fully activated and even down-regulates them. Simple signaling without co-stimulation allows the cell to recognize the primary signal as "self" and not ramp-up responses for future re- sponses as well as the side effects associated with the drug include anaphylactic reactions, respiratory tract infection, urinary tract infections, herpes, elevation in blood pressure, stomach pain, rashes, and systemic injection reactions[13,14].

\section{Brodalumab}

The brand name of the same is Siliq. It is a human monoclonal antibody used for the treatment of inflammatory diseases [15]. The drug shows its action by binding to the interleukin-17 receptor and suppressing the activation of the same [16]. The drug was first approved by FDA in the year 2017. The common side effects associated with the drug include joint pain, headache, fatigue, mouth and throat pain, nausea, muscle pain, and injection site reactions. The recommended dose of the same is $210 \mathrm{mg}$ that is administered as a subcutaneous injection at weeks 0,1 , and 2 followed by $210 \mathrm{mg}$ every 2 weeks [17].

\section{Certolizumab pegol}

The brand name by which the drug is sold is Cimzia. It is a biologic drug that finds its application in the treatment of psoriatic arthritis. It is a monoclonal antibody-based drug that has a specific affinity towards tumor necrosis factor-alpha (TNF- $\alpha$ ) [18]. The drug is manufactured by UCB and got its approval by Food and drug administration in the year 2008. The drug is administered by the subcutaneous route and is available in two options; single-dose vial and single-dose prefilled syringe. Several mild and serious side effects are conferred with the drug. Upper respiratory tract infection, urinary tract infection, rashes, etc. are the few mild side effects observed with the consumption of drug while the serious side effects associated with the same are heart failure, shortness of breath, swollen ankles, fever, fatigue, jaundice, etc. in certain rare instances allergic reactions also precipitate that include swelling in eyelids, lips, hands, mouth, etc. [19,20]

\section{Etanercept}

The brand name of the drug is Enbrel. It is a biologic drug that is used for the treatment of psoriatic arthritis and plaque psoriasis [21]. The drug shows its mechanism of action by interfering with tumor necrosis factors and acts as a TNF inhibitor. The drug is an example of a fusion protein that is developed by recombinant DNA technology, it is made by the combination of naturally occurring soluble human 75-kilodalton TNF receptors linked to an Fc portion of an IgG1. It is a large molecule that binds to TNF- $\alpha$ and decreases its role in autoimmune disorders [22]. The drug is also included in the WHOs list of essential drugs. Etanercept is approved by Food and drug administration in the year 2008. The drug is administered through a subcutaneous route and is available as $0.98 \mathrm{~mL}$ of a $50 \mathrm{mg} / \mathrm{mL}$ solution of etanercept, $0.51 \mathrm{~mL}$ of a $50 \mathrm{mg} / \mathrm{mL}$ solution of etanercept, and $25 \mathrm{mg}$ etanercept [23]. The common side effects observed with the drug include vomiting, nausea, stomach pain, headache, discomfort at 
the site of injection, weakness, heartburn, weight changes, etc. the drug also shows certain serious side effects like bruising, bleeding, swelling, vision changes, chest pain, ongoing cough, etc. [24].

\section{Guselkumab}

Tremfya is the brand name of guselkumab. The drug is an example of a monoclonal antibody product that is found to be effective against plaque psoriasis. It is indicated in the treatment of mild to severe plaque and psoriatic arthritis [25]. The drug got its approval from US FDA in the year 2017 for the treatment of psoriasis. Guselkumab binds with high affinity and high specificity to IL-23, 43 thereby preventing the interaction of the cytokines with its receptor on the surface of the cell. This mechanism is responsible for blocking the initiation of the IL-23 pathway and the subsequent release of other pro-inflammatory cytokines [26]. Since the drug lowers the release of immune system signaling molecules, patients may have more chances of microbial infections. The other common side effects associated with the drug are upper respiratory tract infection, fungal infection, gastroenteritis, herpes simplex infection, etc. the drug is available in the form of the subcutaneous parenteral formulation [27].

\section{Infliximab}

Remicade is the brand name of the drug infliximab, it is a chimeric monoclonal antibody that is used for the treatment of autoimmune diseases. The major complications that are managed by using infliximab include Crohn's disease, ulcerative colitis, rheumatoid arthritis, ankylosing spondylitis, psoriasis, psoriatic arthritis, and Behçet's disease. The drug is formulated as an intravenous injection that is administered to the patient at an interval of six to eight weeks [28]. The drug binds with a high affinity to the TNF- $\alpha$ which is an inflammatory cytokine and prevents its binding with the receptors. The drug has high specificity towards TNF- $\alpha$ and does not bind with TNF- $\beta$ (lymphotoxin). The drug is approved in European Union, United States, and Japan. The side effects like severe infections, reactivation of Hepatitis $\mathrm{B}$, reactivation of psoriasis, druginduced lupus, new-onset vitiligo are commonly observed with the usage of the drug [29].

\section{Ixekizumab}

The brand name of ixekizumab is Taltz. The drug is an example of injectable medication given by the subcutaneous route that is used for the treatment of autoimmune disorders. Ixekizumab is a humanized monoclonal antibody that acts by binding to IL-17A thereby neutralizing and reducing inflammation and also prevents the proliferation of keratinocytes [30]. The drug finds its application in the treatment of moderate to severe plaque psoriasis and is approved in European Union and the United States. The drug is contraindicated in patients suffering from tuberculosis and the preliminary side effects associated with the drug are upper respiratory infections, reaction at the site of injection, and fungal infections [31].

\section{Risankizumab}

The drug is sold under the brand name Skyrizi. It is a humanized monoclonal antibody that targets interleukin $23 \mathrm{~A}$ (IL- $23 \mathrm{~A}$ ) [32]. The drug is approved in European Union, Canada, and the United States and is indicated for the treatment of moderate to severe plaque psoriasis, generalized pustular psoriasis, erythrodermic psoriasis, and psoriatic arthritis especially in cases where an inadequate response is observed with conventional therapy. The drug belongs to the monoclonal IgG1 class antibody; its action is based on binding to the $\mathrm{p} 19$ subunit of IL-23. After binding to the antibody it selectively blocks IL-23 and thus inhibits its pro-inflammatory activity $[33,34]$. It is available in the form of subcutaneous injection. The side effects demonstrated by the drug include fever, chills, sweating, body ache, mouth sore, increased urination, pale skin, unusual bleeding, etc. [35].

\section{Secukinumab}

Cosentyx is the brand name of the drug. The drug belongs to the category of human IgG1к monoclonal antibody. Secukinumab was developed by Novartis and is approved by European Union and the United States for the treatment [36]. The drug is used in the management of psoriasis, ankylosing spondylitis, and psoriatic arthritis. The drug is administered by the subcutaneous route and the formulation is marketed as pre-filled or autoinjectors. Secukinumab inhibits the action of IL-17A that is produced by helper T cells. The inhibition of IL$17 \mathrm{~A}$ results in down-regulation of the same in the plasma of patients suffering from psoriasis and thus blocks the inflammatory reactions and also inhibits the proliferation of keratinocytes [37]. The common side effects observed with the Secukinumab regimen are respiratory tract infection, oral herpes, runny nose, and diarrhea [38].

\section{Ustekinumab}

The drug is sold under the brand name Stelara. The drug belongs to the category of a monoclonal antibody that is used for the treatment of plaque psoriasis and psoriatic arthritis. The drug interferes with the triggering immune responses of the body by binding with interleukins IL-12 and IL-23 and suppresses the release of cytokines [39]. The drug is approved in Europe, the United 
States, and Canada for the treatment of plaque psoriasis. Food and drug administration has approved for use in the treatment of psoriatic arthritis in 2013. The preferred route of administration for the drug is intravenous injection however subcutaneous injections are also available [40]. Posterior reversible encephalopathy syndrome is a risk associated with ustekinumab, the other side effects include upper respiratory infection, headache, and tiredness [41].

\section{Safety concerns associated with biologics}

Although the biologic drugs have demonstrated considerable safety profiles in the clinical trials, however, several drug-specific reactions are associated with biologics [42]. Different classes of drugs show different reactions for instance TNF- $\alpha$ inhibitors and IL-17 inhibitors exhibit serious infections [43], while evidence of adverse reactions with IL-23 inhibitors is not yet reported. Immunogenicity is a matter of concern with biologic regimen since it has a predisposing effect on safety and efficacy the incidence of immunogenicity is more in humanized monoclonal antibodies rather than human monoclonal antibodies a heightened awareness has developed with the progress of the field $[44,45]$. The concerns must be raised while designing a regimen for the management of psoriasis since it is a chronic disease and few patients need to rely on the treatment for the long term, so it is demanded that a longterm observation should be carried out to identify the setbacks with biologics. The safety concerns linked to biologics are represented in figure 2 .

\begin{tabular}{|l|l|l|}
\hline \multicolumn{1}{|c|}{ TNF-a inhibitors } & IL-17 inhibitors & L-23 inhibitors \\
\hline $\begin{array}{l}\text { - Tuberculosis } \\
\text { - Paradoxical } \\
\text { reactions } \\
\text { - Infusion reactions } \\
\text { - Lumps }\end{array}$ & $\begin{array}{l}\text { - Candidiasis } \\
\text { - Neutropenia } \\
\text { - Inflammatory } \\
\text { bowel disease }\end{array}$ & $\begin{array}{l}\text { - No sonificant } \\
\text { adversereactions }\end{array}$ \\
\hline
\end{tabular}

Figure 1: Safety concerns linked to biologics.

\section{Conclusion}

Although biologics represent a newer category of drugs, that is still in an infant stage of development as compared to synthetic drugs. The advantages associated with the usage of biologics like fewer side effects, cost-efficiency, and efficacy against multiple diseases offer an edge over synthetic analogs. Stringent studies regarding the long-term effects of biologics in near future can be relevant in terms of safety and improved patient compliance.

\section{Compliance with Ethical Standards}

The article does not contain clinical studies or patient's data.

\section{Author Contribution}

All authors have made substantial efforts for the compilation of the article. All authors were involved in drafting the article and critical evaluation of the content. All authors have agreed to be accountable for all the aspects of work.

\section{Bibliography}

1. Takeshita J., et al. "Psoriasis and comorbid diseases: Epidemiology". Journal of the American Academy of Dermatology 76 (2017): 377-390.

2. Parisi R., et al. "Global epidemiology of psoriasis: a systematic review of incidence and prevalence". Journal of Investigative Dermatology 133 (2013): 377-385.

3. Crommelin D., et al. "Pharmaceutical evaluation of biosimilars: important differences from generic low-molecular weight pharmaceuticals". European Journal of Hospital Pharmacy (EJHP) 11.1 (2005): 11-17.

4. Declerck PJ., et al. "Biosimilars: controversies as illustrated by rhGH". Current Medical Research and Opinion 26 (2010).

5. Singh JA., et al. "Adverse effects of biologics: a network metaanalysis and Cochrane overview". Cochrane Library: Cochrane Reviews (2011).

6. Mease PJ., et al. "Adalimumab Effectiveness in Psoriatic Arthritis Trial Study Group. Adalimumab for the treatment of patients with moderately to severely active psoriatic arthritis: results of a double-blind, randomized, placebo-controlled trial". Arthritis and Rheumatism 52.10 (2005): 3279-3289.

7. Scheinfeld N. "Adalimumab: a review of side effects". Expert Opinion on Drug Safety 4.4 (2005): 637-641.

8. van de Kerkhof PC. "Consistent control of psoriasis by continuous long-term therapy: the promise of biological treatments". Journal of the European Academy of Dermatology and Venereology 20 (2006): 639-650.

9. Weinblatt., et al. "Adalimumab, a fully human anti-tumor necrosis factor alpha monoclonal antibody, for the treatment of rheumatoid arthritis in patients taking concomitant methotrexate: the ARMADA trial". Arthritis and Rheumatism 48.1 (2003): 35-45.

10. Moreland L., et al. "Abatacept". Nature Reviews Drug Discovery 5.3 (2006): 185-186. 
11. Liu XI., et al. "Monoclonal Antibodies and Fc-Fusion Proteins for Pediatric Use: Dosing, Immunogenicity, and Modeling and Simulation in Data Submitted to the US Food and Drug Administration". Journal of Clinical Pharmacology 59.8 (2019): 11301143.

12. Kumar M., et al. "Pregnancy outcomes following exposure to abatacept during pregnancy". Seminars in Arthritis and Rheumatism 45.3 (2015): 351-356.

13. Porter M L., et al. "Update on biologic safety for patients with psoriasis during pregnancy". International Journal of Women's Dermatology 3.1 (2017): 21-25.

14. Orban T., et al. "Co-stimulation modulation with abatacept in patients with recent-onset type 1 diabetes: a randomised, double-blind, placebo-controlled trial". Lancet 378.9789 (2011): 412-419.

15. Brembilla NC., et al. "The IL-17 Family of Cytokines in Psoriasis: IL-17A and Beyond". Frontiers in Immunology 9 (2018): 1682.

16. Monin L and Gaffen SL. "Interleukin 17 Family Cytokines: Signaling Mechanisms, Biological Activities, and Therapeutic Implications". Cold Spring Harbor Perspectives in Biology 10.4 (2018): a028522.

17. Bauer E., et al. "Brodalumab -an IL-17RA monoclonal antibody for psoriasis and psoriatic arthritis". Expert Opinion on Biological Therapy 15.6 (2015): 883-893.

18. Goel N abd Stephens S. "Certolizumab pegol”. MAbs 2.2 (2010): 137-147.

19. Fleischmann R., et al. "Efficacy and safety of certolizumab pegol monotherapy every 4 weeks in patients with rheumatoid arthritis failing previous disease-modifying antirheumatic therapy: the FAST4WARD study". Annals of the Rheumatic Diseases 68 (2009): 805-811.

20. Smolen J., et al. "Efficacy and safety of certolizumab pegol plus methotrexate in active rheumatoid arthritis: the RAPID 2 study. A randomised controlled trial". Annals of the Rheumatic Diseases 68 (2009): 797-804.

21. Braun J., et al. "Improvement in patient-reported outcomes for patients with ankylosing spondylitis treated with etanercept $50 \mathrm{mg}$ once-weekly and $25 \mathrm{mg}$ twice-weekly". Rheumatology (Oxford) 46.6 (2007): 999-1004.
22. Reich K., et al. "Once-weekly administration of etanercept $50 \mathrm{mg}$ improves patient-reported outcomes in patients with moderate-to-severe plaque psoriasis". Dermatology 219.3 (2009): 239-249.

23. Zhou H. "Clinical pharmacokinetics of etanercept: a fully humanized soluble recombinant tumor necrosis factor receptor fusion protein". Journal of Clinical Pharmacology 45.5 (2005): 490-497.

24. Goffe B. "Etanercept (Enbrel) -- an update". Skin Therapy Letter 9.10 (2004): 1-4.

25. Al-Salama ZT and Scott LJ. "Guselkumab: A Review in Moderate to Severe Plaque Psoriasis". American Journal of Clinical Dermatology 19.6 (2018): 907-918.

26. Chan TC., et al. "Interleukin 23 in the skin: role in psoriasis pathogenesis and selective interleukin 23 blockade as treatment". Therapeutic Advances in Chronic Disease 9.5 (2018): 111-119.

27. Langley RG., et al. "Efficacy and safety of guselkumab in patients with psoriasis who have an inadequate response to ustekinumab: results of the randomized, double-blind, phase III NAVIGATE trial". British Journal of Dermatology 178.1 (2018): 114-123.

28. Flynn JL., et al. "Tumor necrosis factor-alpha is required in the protective immune response against Mycobacterium tuberculosis in mice". Immunity 2 (1995): 561-572.

29. Mohan VP., et al. "Effects of tumor necrosis factor alpha on host immune response in chronic persistent tuberculosis: possible role for limiting pathology". Infection and Immunity 69 (2001): 1847-1855.

30. Atzeni F., et al. "Anti-IL-17 Agents in the Treatment of Axial Spondyloarthritis". Immunotargets Therapy 10 (2021): 141153.

31. Ramaekers BLT., et al. "Ixekizumab for Treating Moderate-toSevere Plaque Psoriasis: An Evidence Review Group Perspective of a NICE Single Technology Appraisal". Pharmacoeconomics 36.8 (2018): 917-927.

32. Blauvelt A., et al. "Efficacy and Safety of Continuous Risankizumab Therapy vs Treatment Withdrawal in Patients With Moderate to Severe Plaque Psoriasis: A Phase 3 Randomized Clinical Trial". JAMA Dermatology 156.6 (2020): 649-658. 
33. Kulig P., et al. "IL-12 protects from psoriasiform skin inflammation". Nature Communication 7 (2016): 13466.

34. Hamza T., et al. "Interleukin 12 a key immunoregulatory cytokine in infection applications". International Journal of Molecular Sciences 11.3 (2010): 789-806.

35. Krueger JG., et al. "Anti-IL-23A mAb BI 655066 for treatment of moderate-to-severe psoriasis: Safety, efficacy, pharmacokinetics, and biomarker results of a single-rising-dose, randomized, double-blind, placebo-controlled trial". The Journal of Allergy and Clinical Immunology 136.1 (2015): 116-124.e7.

36. Rich P., et al. "Secukinumab induction and maintenance therapy in moderate-to-severe plaque psoriasis: a randomized, double-blind, placebo-controlled, phase II regimen-finding study". British Journal of Dermatology 168.2 (2013): 402-411.

37. López-Ferrer A., et al. "Secukinumab (AIN457) for the treatment of psoriasis". Expert Review of Clinical Immunology 11.11 (2015): 1177-1188.

38. Blauvelt A. "Safety of secukinumab in the treatment of psoriasis". Expert Opinion on Drug Safety 15.10 (2006): 1413-1420.

39. Trinchieri G. "Interleukin-12 and the regulation of innate resistance and adaptive immunity". Nature Review on Immunology 3.2 (2003): 133-46.

40. Xu L., et al. "Cutting edge: regulatory T cells induce CD4+CD25Foxp3- T cells or are self-induced to become Th17 cells in the absence of exogenous TGF-beta". Journal of Immunology 178.11 (2007): 6725-6729.

41. Fishwild DM., et al. "High-avidity human IgG kappa monoclonal antibodies from a novel strain of minilocus transgenic mice". Nature Biotechnology 14.7 (1996): 845-851.

42. Dalgard FJ., et al. "The psychological burden of skin diseases: a cross-sectional multicenter study among dermatological outpatients in 13 European countries". Journal of Investigative Dermatology 135 (2015): 984-991.

43. Kurd SK., et al. "The risk of depression, anxiety, and suicidality in patients with psoriasis: a population-based cohort study". Archives of Dermatology 146 (2010): 891-895.

44. Dowlatshahi EA., et al. "The prevalence and odds of depressive symptoms and clinical depression in psoriasis is patients: a systematic review and meta-analysis". Journal of Investigative Dermatology 134 (2014): 1542-1551.
45. Singh S., et al. "Psoriasis and suicidality: A systematic review and meta-analysis". Journal of the American Academy of Dermatology 77 (2017): 425-440.

\section{Volume 4 Issue 10 October 2021}

(C) All rights are reserved by Shashi Kiran Misra., et la. 